\title{
Development and Application of Dynamic Timing Optimization Platform for Big Data Intelligent Traffic Signals
}

\author{
Zhao Wang ${ }^{1 *}$, Mengjie Wang ${ }^{2}$ and Wenqiang $\mathrm{Bao}^{3}$ \\ ${ }^{1}$ Big Data Research Center, Jianghan University, Wuhan, Hubei, 430056, China \\ ${ }^{2}$ Big Data Research Center, Jianghan University, Wuhan, Hubei, 430056, China \\ ${ }^{3}$ YiBen Investment Fund, New York, 10005, United States
}

\begin{abstract}
As the number of car ownership increases, road traffic flow continues to increase. At the same time, traffic pressure at intersections is increasing as well. At present, most of the traffic lights in China are fixed cycle control. This timing control algorithm obviously cannot make timely adjustments according to changes in traffic flow. In this case, a large number of transportation resources would be wasted. It is very necessary to establish a dynamic timing system for Big data intelligent traffic signals. In this research, the video recognition method was used to acquire the number of vehicles at the intersection in real time, and the obtained data was processed by the optimization algorithm to make a reasonable dynamic timing of the traffic signals. The test results show that after using the big data intelligent traffic signal dynamic timing optimization control platform, in the experimental area, the overall total delay time was reduced by $23 \%$, and the travel time was reduced by $15 \%$. During the off-peak period, the overall total delay time in the experimental region was reduced by $17 \%$ and travel time was reduced by $10 \%$. The big data intelligent traffic signal dynamic timing optimization platform would improve the operational efficiency and traffic supply capacity of the existing transportation infrastructure, and could provide real convenience for citizens.
\end{abstract}

\section{Introduction}

The city's transportation network is as complex as a transmission network. The crossover capacity of transmission equipment is one of the important influence indicators of transmission efficiency. Similarly, the traffic efficiency of urban traffic intersections is also an important factor affecting urban traffic conditions. According to the research, compared with other optimization methods, such as road reconstruction and traffic flow prediction, the simple, most direct and effective optimization method without affecting daily traffic operation is to improve the traffic efficiency of traffic intersections by optimizing the signal timing scheme.

With the increase of car ownership, there is often such a phenomenon at the intersection of urban main roads and non-main roads that many cars parked in the traffic direction of the red light. However, there are very few vehicles in the green light traffic direction. The serious waste of transportation resources like that has caused the pressure at the intersection to become larger and larger. The existing signal light control systems are mainly divided into two categories: timing control and inductive control. On the one hand, timing control cannot adapt to the dynamic change of traffic flow. On the other hand, inductive control is susceptible to external interference. Therefore, it is very important to establish other reasonable intelligent traffic control technologies.
Intelligent traffic signal timing system based on video recognition can make rational use of intersection resources. With the help of this new timing system, the intelligent distribution of traffic flow could be realized, and the traffic capacity of the intersection could be maximized.

\section{Domestic and foreign signal light research status}

The core function of the intelligent transportation project platform based on big data is the control of traffic signals. In worldwide, traffic signal control is divided into three stages. The first stage is the emergence of traffic light controllers. In 1868, the first traffic light in history was installed in London, England. The second stage is the urban signal light system realizes multi-junction coordination and unified decision-making. In the 1950s and 1960s, the development of electronic computers greatly promoted the development of the urban signal lights. The third stage is the integration of artificial intelligence into the application of urban signal light system. At present, urban traffic signal systems incorporate artificial intelligence. The main technologies include the application of advanced theoretical results such as artificial neural networks, fuzzy logic and genetic algorithms in signal light systems.

Although artificial intelligence has been applied in the field of traffic signal control, domestic related 
scientific and technological theoretical researches are still relatively backward, and there are only a few regulations issued by the Ministry of Public Security. Most cities solve their traffic problems by purchasing foreign commercial systems. More widely used such as the following foreign systems:

Table 1. Foreign traffic signal control system.

\begin{tabular}{cc} 
Country & System \\
\hline UK & SCOOT(Split-Cycle-Offset Optimization Technique) \\
\hline \multirow{2}{*}{ US } & TRANSYT-7F(Traffic Network Study Tool) \\
\cline { 2 - 2 } & Passer II \\
\cline { 2 - 2 } & Synchro \\
\hline
\end{tabular}

Australian $\quad$ SCATS(Sydney Coordinated Adaptive Traffic System)

The characteristics of domestic and foreign urban traffic are different. The number of non-motorized vehicles in China is larger than that of pedestrians. China's urban concentration is large. Foreign systems may not be in line with China's traffic conditions. On the other hand, such systems are sold at high prices without exception. Due to trade secrets, users cannot understand in depth, resulting in unsatisfactory results in practical applications. Therefore, the localization of urban signal light systems is imperative.

\section{Principles of the system}

The intelligent traffic signal optimization control platform automatically recognizes the vehicle traffic information in the road traffic video through the highdefinition camera installed at the intersection and the periphery. Convert unstructured image data into structured data and store the generated database. Applying the big data deep mining technology, the traffic information of the optimized signal light could be obtained through the analysis of the multi-source traffic big data. The technology can extract stable regional traffic operation rules from a large amount of historical data, and accurately predict upcoming traffic conditions based on real-time traffic information. Through the machine deep learning function, the system will selflearn through feedback information, and constantly improve the timing algorithm to ensure that the design of each cycle is based on the optimal timing scheme under current information.

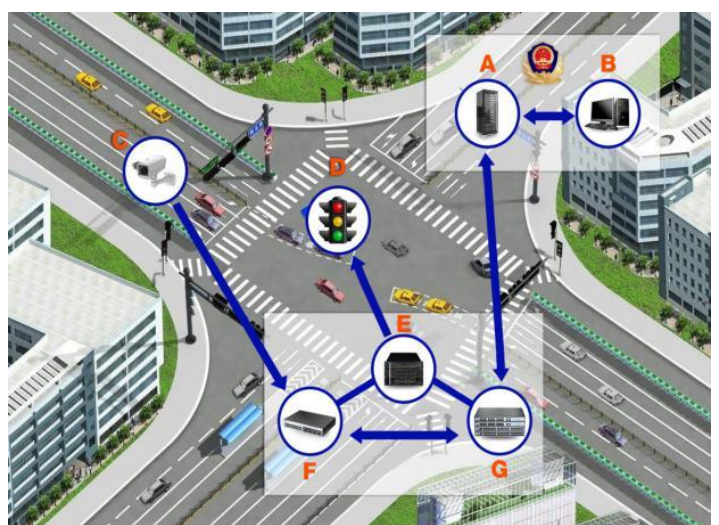

- A: Data storage and processing server

- C: Monitoring

- E: Intelligent controller

- F: video processing module
- G: Communication module

- $\mathrm{A} \rightleftharpoons \mathrm{G}$ : wired or wireless data transmission

- $\mathrm{C} \rightarrow \mathrm{F}$ : wireless video transmission

- $\mathrm{E} \rightarrow \mathrm{D}$ : real-time control

Figure 1. System Working Principle.

\subsection{Data collection}

\subsubsection{Upstream arrival information}

The upstream detector of the inlet can provide the moment when the vehicle reaches the detection location. For example, if $\mathrm{n}$ vehicles pass the detection position within a certain period of time, the detector can record the elapsed time of the $n$ vehicles, a1, a2, a3, ..., an.

\subsubsection{Departure information}

The entrance lane detector can provide the moment when the vehicle leaves the parking line. For example, if there are $\mathrm{m}$ vehicles passing through the parking line in a certain period of time, the detector can record the elapsed time of the $m$ vehicles, $d 1, d 2, d 3, \ldots, d 4$.

\subsubsection{Ideal driving time}

Each upstream detection location corresponds to an ideal travel time $\mathrm{t}$, which means how much time the vehicle passing through the detection location needs to leave the parking line without experiencing delays. The value is calculated by dividing the distance $\mathrm{d}$ from the upstream detection position to the stop line by the ideal speed s of the road segment.

\subsection{Delay estimation method}

If a total of $X$ vehicles pass and leave an entrance lane within a certain period of time, the total delay of all 
vehicles on the entrance lane is $(\Sigma$ $\left.d i X i=1-\sum a i X i=1-X t\right)$. And:

$\mathrm{d}_{\mathrm{i}}=$ the departure time of the $\mathrm{i}$-th vehicle leaving the entrance lane during this time period;

$a_{i}=$ the time upstream of the $i$-th vehicle passing the upstream detection position of the inlet lane during the time period;

$\mathrm{t}=$ ideal driving time for the detection position upstream of the inlet.

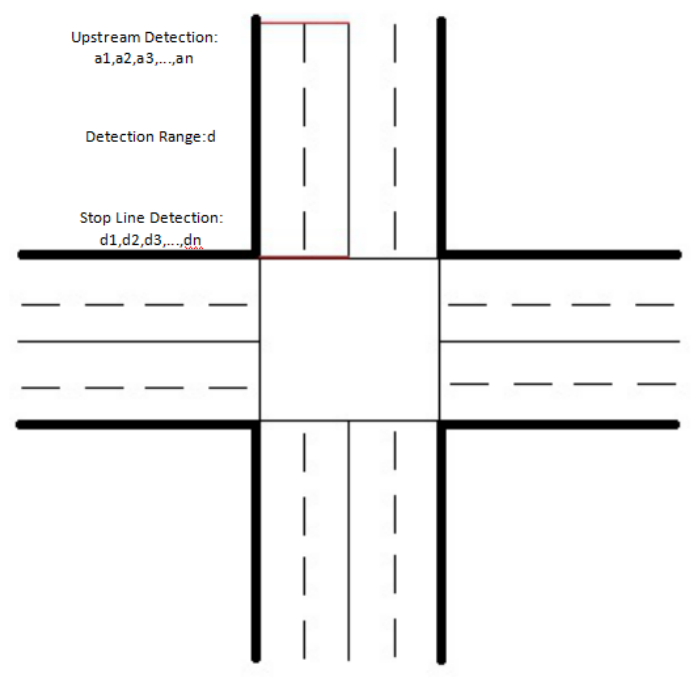

Figure 2. Detector LOCATION.

\subsection{Method principle}

The above delay estimation method is applicable to the upstream detection position and the case where there is no other entrance or exit between the parking lines. It means that the vehicle passing the upstream detection position will leave the parking line. To illustrate the principle of the method, three vehicles are taken through this entrance road as an example to discuss the two situations separately:

Case 1: The vehicles pass through the upstream detection position and the parking line in order. In this case $a_{1}$ corresponds to $d_{1}, a_{2}$ corresponds to $d_{2}, a_{3}$ corresponds to $\mathrm{d}_{3}$.

In this situation, the car 1 (the first vehicle that arrives at the upstream position and the first one leaves the parking line) should ideally leave the parking line at

$\left(a_{1}+t\right)$ and actually leave at $d_{1}$. Therefore, the delay experienced is $\left(d_{1}-a_{1}-t\right)$. And so on, the delays experienced by car 2 and car 3 are $\left(d_{2}-a_{2}-t\right)$ and $\left(d_{3}-a_{3}-t\right)$, respectively. Therefore, the total delay of the three cars is $\quad\left(\sum_{i=1}^{3} d_{i}-\sum_{i=1}^{3} a_{i}-3 t\right)$.

Case 2: Because of different lanes, overtaking or lane change, $a_{1}$ corresponds to $d_{2}, a_{2}$ corresponds to $d_{3}, a_{3}$ corresponds to $d_{1}$.

In this case, the delays of the three vehicles are $\left(d_{2}-a_{1}-t\right),\left(d_{3}-a_{2}-t\right)$ and $\left(d_{1}-a_{3}-t\right)$, respectively. Therefore, the total delay of the three cars is also $\left(\sum_{\mathrm{i}=1}^{3} \mathrm{~d}_{\mathrm{i}}-\sum_{\mathrm{i}=1}^{3} \mathrm{a}_{\mathrm{i}}-3 \mathrm{t}\right)$.

From the discussion above, it has demonstrated that the method is applicable regardless of whether the vehicle passes through the upstream detection position and the stop line in order (not affected by the number of lanes, overtaking, lane change, etc.). Besides, since the change of delay time when using different timing schemes is one of the research observations, there is no requirement for the estimation accuracy of $t$. In the case of a large amount of data, there are a small number of vehicles in the upstream detection position and the entry and exit between the parking lines. More accurate delay change values can be estimated

\subsection{Traffic volume during peak hours}

Traffic volume refers to the amount of traffic passing through an intersection during a certain period. During the peak period, people hope that more vehicles can be used in a unit of time to improve traffic efficiency and increase traffic capacity. Therefore, the traffic volume is an important indicator for evaluating the pros and cons of the signal timing scheme during peak hours. The traffic volume of each flow direction could be collected through video data. It could also aggregate indicators such as the import road traffic volume, intersection traffic volume and road network traffic volume.

\section{System advantages}

This software can dynamically generate regional timesegment segmentation and timing schemes based on traffic time-sharing data. The software uses the most advanced dynamic data-based traffic optimization algorithm, combined with deep learning and evolutionary algorithms, to accurately predict the number of times the vehicle has passed, thus generating a constantly selfimproving phase setting, timing, and phase difference. This system is a regional mesh optimization different from the previous technical solutions. It is to optimize the overall delay time of the entire optimized area. At the same time, the software can also manually set the road with priority, thus achieving green wave access. When the input data is missing due to an accident, the software can also generate a suboptimal timing scheme based on historical data and self-check. Therefore, it has good fault tolerance and robustness.

\subsection{Video capture data}

At present, most of the domestic induction coils are used to detect vehicle flow data. Due to urban road construction, the induction coils are frequently damaged. The intelligent traffic signal optimization control platform is based on the external field video image data acquisition, and the data acquisition is more stable.

\subsection{Not relying on traditional traffic models}

The traditional traffic models are derived from foreign theoretical systems and do not meet the current domestic traffic conditions, resulting in large errors in traffic flow prediction. The new machine deep learning algorithm of the intelligent traffic signal optimization control platform finds the law from the real data, does not depend on the 
traditional traffic model, and can be well adapted to the domestic actual traffic conditions.

\subsection{Multi-channel collaboration}

To solve the urban traffic problem can not only focus on the traffic itself, but from the entire large transportation system, the whole society to consider, and more consideration of control and management.

\subsection{Algorithm optimization}

Through the average delay time of big data operation in different schemes, through the massive calculation, the set of time-matched data with the lowest average delay time and the highest traffic volume is selected as the optimal timing scheme.

\section{Research Result}

Through the intelligent traffic signal optimization control platform, the Wujiashan area in the Dongxihu District of Wuhan City was reconstructed. Eight road intersections in the area were selected, and the regional traffic signal control model was established according to the road network geometry, lane setting, traffic control and flow information. The intelligent traffic signal optimization control platform could optimize the traffic signal control model based on the actual situation after researching and analysing the model. And it could provide real-time solutions to road emergencies. The ratio of the overall flow rate $\mathrm{v}$ to the capacity $\mathrm{c}(\mathrm{v} / \mathrm{c})$ for peak hour and offpeak hours was approximately equal to 1 and 0.6 , respectively. The test results show that after using the intelligent traffic signal optimization control platform, the overall total delay time of the region during the peak period was reduced by $23 \%$, and the travel time was reduced by $15 \%$. During the off-peak period, the overall total delay time in the region was reduced by $17 \%$ and travel time was reduced by $10 \%$. It can be seen that the intelligent traffic signal optimization control platform has more obvious improvement on the road junctions during peak periods.

\section{Conclusion}

The intelligent traffic signal optimization control platform could improve the operational efficiency and traffic supply capacity of the existing transportation infrastructure through the implementation of DITS simulation prediction and self-learning algorithms and the implementation of big data analysis platforms. In the direction of traffic demand, it is possible to improve the spatial and temporal distribution characteristics of traffic demand, and "cut the peaks and fill the valley". The contradiction between traffic demand and transportation supply has been alleviated. And massive data could be real-time screened and analysed. This system could not only effectively provide urban managers with quantitative basis and standards for decision-making, but also provide tangible convenience for citizens to travel.

\section{References}

1. Akhan, A. (2015) Mobile Standards-Based Traffic Light Detection in Assistive Devices for Individualswith Color-Vision Deficiency. IEEE Transactions on Intelligent Transportation Systems.

2. Chen, T., Wang, Y., Xiao, C. (2016) A machine vision apparatus and method for can-end inspection. IEEE Transactions on Instrumentation and Measurement.

3. Jang, K., Kim, H., Jang, I.G. (2015) Traffic signal optimization for over-saturated urban networks:queue growth equalization. IEEE Transactions on Intelligent Transportation Systems.

4. Seyed, A.F., Ardalan, V. (2016) Crowdsourcing Phase and Timing of Pre-Timed TrafficSignals in t-he Presence of Queues:Algorithms and BackEnd System Architecture. IEEE Transactions on Intelligent Transportation Systems.

5. Zhao, J., Li, W., Wang, J. (2016) Dynamic traffic signal timing optimization strategy incorporating various vehicle fuel consumption characteristics. IEEE Transactions on Vehicular Technology.

6. Fadi, M., Rachid, B., Abdellah E. M. (2011) A dissipativity-based approach to traffic signal control for an over-saturated intersection. Journal of the Franklin Institute. 